\title{
THE ESCAPE OF DIPHTHERIA BACILLI INTO THE BLOOD AND TISSUES. ${ }^{1}$
}

\author{
By A. A. Kanthack and J. W. W. Stephens. \\ From the Pathological Laboratory, St. Bartholomew's Hospital.
}

WHILE bringing the following observations ${ }^{2}$ before the Society, we are conscious that the escape of the diphtheria bacillus into the blood, tissues, and more or less distant organs from the seat of infection, has already been made the subject of careful investigation by others, especially by Continental and American writers. In this country, however, the researches bearing on this question have received but little attention, although it is one both of theoretical and practical importance. We venture, therefore, to give a short review of our own observations. Before doing so, we shall briefly consider the work already done.

Wright ${ }^{3}$ of Boston, in a long and exhaustive paper, embodying a large amount of work, states that in 14 fatal cases he found, by cultivation on blood serum, diphtheria bacilli-

In the lungs, on 13 occasions ;

In the cervical or bronchial glands, on 5 occasions;

In the liver, on 3 occasions;

In the spleen, twice;

In the mesenteric glands, twice;

In the heart's blood, once;

And in the kidney, once.

He also notices the escape of the bacillus into the stomach and œsophagus.

Since then Wright has published another paper in conjunction with Stokes, ${ }^{4}$ from which we gather that in 31 fatal cases of diphtheria, which apparently include the above 14 cases, the Klebs-Löffler bacillus occurred-

${ }^{1}$ Communicated to the Pathological Society of London, 21st January 1896, and published with permission of the Council.

2 To make our observations complete we have incorporated in this paper several cases which have occurred since this communication was made.

${ }^{3}$ Boston Med. and S. Journ., October 1894.

${ }^{4}$ Ibid., March 21, 28, and April 4, 1895. 
In the lungs, 30 times;

In the kidney, 6 times;

In the lymphatic glands often;

In the liver, 9 times (this viscus was examined in 29 cases);

In the spleen, 5 times ;

In the heart's blood and in other regions, occasionally.

Frosch ${ }^{1}$ had demonstrated the presence of the diphtheria bacillus, by means of cultivation, in the internal organs in 10 out of 15 fatal cases, most frequently in the broncho-pneumonic foci, in the spleen, the cervical and bronchial glands.

Kolisko and Paltauf ${ }^{2}$ found it in the spleen, Schmorl ${ }^{3}$ in the cervical glands in 7 cases out of 10 .

Booker ${ }^{4}$ separated it from the spleen, submaxillary gland, lungs, and heart's blood in a single case examined.

Johnston ${ }^{5}$ examined a single case and found it in the lungs, while Strelitz ${ }^{6}$ saw it in the lung in 1 case out of 8 , and Flexner ${ }^{7}$ in 1 case out of 2 .

Kutscher ${ }^{8}$ was able to demonstrate the diphtheria bacillus microscopically in lung sections, and in part by means of cultivations in 8 cases out of 9 examined, and once also in the kidney and in the liver; and Canon ${ }^{9}$ found it repeatedly in cadaveric blood.

Recently Stokes ${ }^{10}$ has examined 9 fatal cases of diphtheria which had been treated with antitoxine, by means of cultures taken on serum from the lung, spleen, kidney, and heart's blood. In all cases the $B$. diphtherioe was found in the lungs, in 4 cases also in the kidney, in 1 case in the spleen, and in another in the heart's blood.

To this last we must add the observations made by Sims Woodhead, ${ }^{11}$ to which he alluded in last year's discussion on diphtheria. "So far as he could make out from his notes of about 50 post-mortem examinations, in only 5 instances had he been able to find the bacillus in the lung." At the same meeting one of us ${ }^{12}$ showed microscopic sections from 2 cases, exhibiting the presence of the Klebs-Loffler bacillus in the smallest bronchioles and in the broncho-pneumonic foci.

It is evident, then, that in fatal cases of diphtheria the escape of this bacillus from the seat of infection is by no means uncommon, and that the general statement that "the bacillus diphtheriæ is found

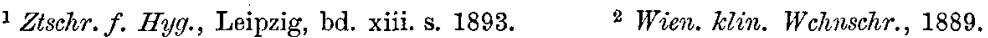

${ }^{3}$ Schmidt's Jahrb., Leipzig, bd. cexxxvii. p. 110.

4 Arch. Pediat., N.Y., 1893, vol. x. $\quad{ }^{5}$ Montreal Med. Journ., Sept. 1891.

${ }^{6}$ Arch. f. Kinderh. Stuttgart, 1891, bd. xiii.

7 Johns Hopkins Hosp. Bull., Baltimore, 1893.

${ }^{8}$ Ztselkr, f. Hyg., Leipzig, bd. xviii. s. 175 .

${ }^{9}$ Deutsche med. Wehnschr., Leipzig, 1893, s. 1039.

${ }^{10}$ Boston Med. and S. Journ., Dec. 12, 1895.

11 Trans. Path. Soc. London, 1895, vol. xlvi. p. $311 . \quad 12$ Ibid. p. 304.
} 
in the false membrane and in the membrane alone," and that "it does not enter the lymphatic or blood stream," ${ }^{1}$ is by no means correct.

1. Passing now to our own observations, which were begun in January 1895, we have found that in the cervical and bronchial lymphatic glands diphtheria bacilli are often found in fatal cases. Unfortunately we kept no regular account of the frequency of the secondary lymphatic infections, and cannot, therefore, give a figure; but we venture to say that it is of common occurrence.

2. In 26 recent fatal cases we carefully examined the lungs for the presence of diphtheria bacilli-of earlier cases no systematic consecutive examination was made, so that these must be disregarded for statistical purposes. Films were always made and stained with anilinegentian-violet, and cultivations on a special form of serum agar-agar were employed. In all these $26^{\circ}$ cases diphtheria bacilli were obtained with ease and in large numbers, and in most cases it was not difficult to detect them in the films.

3. In 4 of these 26 fatal cases the spleen had not been examined, in a fifth case the agar-agar had been insufficiently sterilised, but in the remaining 21 cases it contained diphtheria bacilli in 10 instances. For the purpose of separating the bacillus from the spleen, the surface of the organ was cauterised with a red-hot glass rod, and with a platinum loop a little pulp was removed, and 2 to 4 tubes inoculated therewith.

4. The kidneys were examined for the presence of diphtheria bacilli on three occcasions: the latter were dicovered twice.

The serum agar-agar which we use is prepared with ascitic, pleuritic, or hydrocele fluid, to which we add enough caustic potash to prevent it from coagulating on boiling. We then mix this alkaline fluid with agar-agar (1.5 to 2 per cent.), and boil it up in the ordinary way, and finally, after filtration, add to the clear agar-agar 5 per cent. glycerine. This is by far the best medium for the separation of the diphtheria bacillus with which we are acquainted. Its mode of preparation has recently been published.2 We consider that our results are greatly due to the possession of a good selective nutrient medium.

We see, then, from these results that the diphtheria bacillus escapes most readily into the lungs, and is there present in large numbers, for, contrary to Frosch and Wright, for the purpose of cultivation we used small quantities of material for inoculation; and we cannot follow Stokes in his argument that in these situations the bacilli are only found in small numbers, for if we remember that every platinumloopful spread on the agar-agar generally gave rise to numerous colonies, it must be evident that the whole organ contained an

1 Sidney Martin, Rep. Med. Off. Privy Council, London, 1892-93, p. 425.

${ }^{2}$ Lancet, London, 1896, vol. i. p. 835. 
appallingly large number of diphtheria bacilli. On comparing our figures with those obtained by Kutscher, Wright, and Stokes, we find a pretty close agreement, so far as the lungs are concerned, for they obtained bacilli from the lungs in 47 out of 49 cases, while we obtained them in all our 26 cases. It is evident, then, that in fatal cases of diphtheria the specific organism is very frequently indeed found in the lungs; if we add our cases to those described by the Americans, in 65 instances out of 66 . We should mention, however, that although Wright says in the summary of his first paper that the lungs contained the Klebs-Löfller bacillus in 13 out of 14 cases, from his published notes we could only collect 12 cases which showed the diphtheritic infection in the lungs, so that possibly there is an omission somewhere in his notes.

We must now examine the post-mortem conditions of the lungs in these cases more closely.

(a) In Wright's 13 cases of diphtheritic lung infection, described in his first paper, broncho-pneumonia existed in 10, in our cases in 15, so that we must take exception to the statement frequently made that the broncho-pneumonia in diphtheria is of pyococcal, or more especially of streptococcal, origin. In most cases it appears to be a direct diphtheritic complication.

In those cases which macroscopically presented nothing abnormal, possibly subsequent histological investigation will show either the earliest stages of broncho-pneumonia or the changes of so-called capillary bronchitis.

Another point which our observations bring out is the fact that acute broncho-pneumonia complicating diphtheria is not necessarily or even generally a foreign infection. There is a distinct tendency amongst bacteriologists to regard the streptococcus more than any other organism as the cause of such broncho-pneumonia. This view seems to us to be as harmful as it is incorrect.

(b) The next point to be considered is the nature and type of the diphtheritic lesion. We find that in Wright's 13 cases above mentioned it extended into the larynx in 9 cases, and in our 26 cases 24 times. It further appears that broncho-pneumonia is most frequently associated with membrane in the larynx, for in 13 out of the 24 laryngeal cases of ours there was broncho-pneumonia; in the other 11 cases the lung is described in 5 cases as natural, in 5 as collapsed, in 1 as tubercular. In one case there was bronchopneumonia, but neither during life nor post-mortem was there membrane either in the fauces or in the larynx, but diphtheria bacilli were readily obtained after death from the mucous membrane of the trachea, so that probably this case also was one of laryngeal diphtheria. Amongst the 9 cases of laryngeal diphtheria incorporated in Wright's paper, broncho-pneumonia was found after death in 6 , in the other 3 cases the lung is described in 1 case as containing an 
infarction, in another as ecchymosed, and in the third as congested.

(c) Tracheotomy had been performed in 18 out of our 26 cases, in one of these after previous intubation.

In Wright's cases no mention, unfortunately, is made regarding this point. In 12 of our tracheotomy cases there was also bronchopneumonia, but from the clinical history we cannot possibly accuse the operation of being the cause of the lung affection, because in most cases the tracheotomy was an emergency operation, since the children were generally admitted in a dying or serious condition. So far as we have been able to obtain detailed notes we find that in most instances tracheotomy was performed on, or soon after, admission, and that the patients rarely survived the operation longer than 1 to 3 days.

From our own experience, then, we cannot agree with Sims Woodhead in the assumption that micrococci in the pneumonic patches are most frequently associated with tracheotomy. In all fairness to Dr. Woodhead, it should be remarked, however, that he makes this statement with due reservations.

That the broncho-pneumonia is not caused by the tracheotomy is further shown by the fact that in 3 cases there was broncho-pneumonia without a previous tracheotomy, and in 1 of these there was membrane in the trachea.

Again, we have 6 cases of tracheotomy without obvious bronchopneumonia. Hence, judging from our own observations, we come to the conclusion that the broncho-pneumonia, which is so frequently met with in fatal cases of diphtheria, is often, if not generally, of diphtheritic nature, and is, as a rule, associated with or preceded by laryngeal diphtheria. We are quite aware of the fact that the KlebsLoffler bacillus is never found as a pure growth in the lung, and that it is generally accompanied by streptococci and other micrococci. These organisms, however, are also found at the primary seat of infection, and are there only of secondary importance, and there is no reason why in a broncho-pneumonia where the diphtheria bacillus is present in large numbers they should be of greater importance. Moreover, it must be kept in mind that staphylococci, pneumococci, and streptococci are normal inhabitants of the bronchi, bronchioles, and alveoli; and therefore on cultivation must of necessity appear on the agar-agar surface. Microscopically the diphtheria bacilli can frequently, if not generally, be seen in the alveolar spaces. Since our own histological investigations are as yet incomplete we are compelled to speak with some caution.

Wright and Stokes agree with us that the broncho-pneumonia of diphtheria may be due to the diphtheria bacillus, with or without the association of streptococci, staphylococci, and pneumococci. In 19 cases of broncho-pneumonia complicating diphtheria, they found the $B$. diphtherio alone in 8 ; together with the streptococcus in 5 ; 
together with the streptococcus and the staphylococcus aureus in 2 ; together with the streptococcus, pneumococcus, and staphylococcus aureus in 1 ; together with the staphylococcus aureus in 1 ; together with the streptococcus and pneumococcus in 1 ; and the streptococcus alone in 1 case. On the other hand, as we also observed, the $B$. diphtherice may be found with or without these micrococci without apparently there being any broncho-pneumonia.

In our studies a small platinum-loopful is removed from the lungs and smeared over the surface of the agar-agar, and the fact that numerous, or even any diphtheria bacilli colonies are obtained must mean that the lungs are crowded with them, and that the amount of toxines produced in them must be enormous. We shall return to this point.

(d) Spleen.-Wright succeeded in finding bacilli in the spleen in 2 cases out of 14 . In 5 cases the condition of the spleen, postmortem, is described as one of acute congestion, but in the 2 cases where the bacilli were found no remark is made as to its condition.

In one of those cases bacilli were also present in the lungs, though in the other case the examination of the lungs gave negative results. Wright and Stokes, adding all their cases together, found the diphtheria bacillus in the spleen in 6 out of 40 cases.

In our 26 cases bacilli were found in the spleen in 10 cases, in 4 of the remaining 16 cases no examination was made, and in 1 the examination was unsuccessful on account of insufficient sterilisation, so that we may say that bacilli were looked for in 21 cases and found in 10. (This result, differing from that of Wright and Stokes, we think, may be due to chance or to the use of our particular serum agar-agar.)

The condition of the spleen is not noted in 15 cases, and is of little importance.

We find, further, that the presence of bacilli in the spleen is associated in all these cases with the presence of bacilli in the lungs, and that in 70 per cent. there was broncho-pneumonia.

We conclude, therefore, that in fatal cases of diphtheria the KlebsLöffler bacillus is far more frequently found in the lungs and spleen than is generally supposed; in the lungs, indeed, it is almost always present in large numbers. We do not venture to generalise from these observations, which cannot be considered numerous, but if we add to them those of others, especially those of Wright and Stokes, they gain considerably in importance, and go far towards proving our thesis "that in fatal cases there is an extensive escape of the diphtheria bacillus into the lungs and other organs," that is, "that in fatal cases the diphtheritic infection readily becomes general."

If we now review all the facts in our possession we find that-

1. The diphtheria bacilli may escape from the original seat of infection by direct transference, as, for instance, when we have 
cutaneous diphtheritic sores during the course of diphtheria. We have had two such cases at St. Bartholomew's Hospital, where children admitted for very severe diphtheria also presented several cutaneous membranous sores in the face and on the neck, in which the KlebsLöffler bacillus was readily found. Wright also describes several such cases.

2. The bacilli may escape along existing passages from the seat of infection-

(a) To the nose;

(b) To the eyes;

(c) To the ears;

(d) To the pharynx and oesophagus ;

(e) To the stomach and intestines;

(f) To the trachea bronchi, bronchioles, and lung alveoli.

3. The bacilli may escape from the seat of infection along the lymphatics-

(a) Into the cervical glands;

(b) Into the bronchial glands.

4. The bacilli may escape from the primary or secondary seat of infection through the circulation--
(a) Into the blood (heart);
(b) Into the spleen;
(c) Into the liver;
(d) Into the kidney.

The occasional presence of the bacilli in the mesenteric glands may be explained in various ways. They may have escaped there either

(a) Through the circulation; or

(b) From the stomach or intestines, infected secondarily; or

(c) Through the digestive tract, having been swallowed, without leading to a secondary infection.

Wright, Abbott and Ghriskey, ${ }^{1}$ and Zarniko ${ }^{2}$ have recorded animal experiments which clearly establish the possibility of this bacterial escape, although, as our experiments also show, it does not seem to be of frequent occurrence.

Thus Wright finds the diphtheria bacillus amongst inoculated animals-

In 155 livers, 19 times;

In 152 spleens, 15 times;

In 153 blood examinations, 7 times; and

In 151 kidney examinations, 4 times.

Abbott and Ghriskey demonstrated that in exceptional instances diphtheria bacilli, when deposited under the skin of smaller animals (guinea-pigs), may be found in the lymphatic apparatus of the omentum, and that when the injections are made into the testicles of these animals they are also found in masses in the omental lymph spaces of 3 out of 4 animals inoculated. To these experiments also

${ }^{1}$ Johns Hopkins Hosp. Bull., Baltimore, April 1893.

${ }^{2}$ Centralbl. f. Bakteriol. u. Parasitenk., Jena, 1889, bd. vi. 
must be added those of Klein, ${ }^{1}$ who has shown that on inoculating milch cows subcutaneously, diphtheritic lesions may appear in distant areas and diphtheria bacilli may escape with the milk.

Our own experiments on this point are far from complete, and we shall therefore, for the present, abstain from any criticism of these observations of others.

We now pass to the final conclusions, which we venture to draw from these investigations.

1. They have first a direct bearing on the process of intoxication in diphtheria. Sidney Martin ${ }^{2}$ explains this process in the following manner. His major premise is that the diphtheria bacillus is not found beyond the membrane or primary seat of infection. Here a ferment or enzyme-like substance is secreted or formed by the bacillia substance possessing strong proteolytic action. This body is absorbed into the blood and tissues, and by virtue of its digestive action on the tissue proteids leads to the formation of specific toxic albumoses- the diphtheria albumoses; so that then, according to him, the primary ferment-like product of the bacteria is not the actual toxine, but the intoxication is brought about by the albumoses and organic acid called into existence by this ferment. This view is based on the observation, that from the blood and organs, and especially from the spleen of children which have succumbed to diphtheria, large quantities of albumoses can be obtained, which, when injected into the rabbits, produce most striking diphtheritic lesions. From cultures in alkalialbumen solutions, albumoses acting similarly could be obtained. In the membrane, however, albumoses were practically absent.

This view seems to us to be open to some objections-

1. We have no evidence that the ferment-like substance when inoculated into an animal leads to the formation of toxic albumoses.

2. The ferment-like body of the membrane is much more active than the albumoses from the spleen and organs.

3. Evidence is gradually accumulating that the specific toxines are not albumoses or ordinary proteids, and in the case of the diphtheria bacillus by growing it in asparagin solution, Uschinsky ${ }^{3}$ has obtained a toxine which does not react as an albumose or ordinary proteid.

We know that albumoses are formed in the course of many infective processes, but that, as in the case of tetanus, they are apparently mere concomitants or contaminations, which, during the precipitation with alcohol or whatever reagent is used, may be carried down together with the true poison.

We believe that the toxine is a direct product of the diphtheria bacillus, which in the membrane is found in its purest form, and that wherever the diphtheria bacillus occurs there also this toxine exists.

${ }^{1}$ Rep. Med. Off. Privy Council, London, 1889, p. 167 ; ibid., 1890, p. 219.

${ }^{2}$ Ibid., 1892-93, pp. 427-430.

${ }^{3}$ Centralbl. f. Bakteriol. u. Parasitenk, Jena, bd. xiv. p. 316. 
Hence, since this bacillus exists in the lungs in large numbers in fatal cases, the toxine also must exist here in great quantity. Therefore, in such cases the amount of poison present at death is very considerable. It is absorbed as such by the blood and tissues, and passes therefore also into the spleen and kidneys. Add to this that we frequently find diphtheria bacilli actually in the spleen, and we can no longer wonder that toxic substances occur in this organ. That in precipitation with alcohol they appear to be albumoses may be accidental, the albumoses coming down together with the true toxine, so that as a matter of fact we have a mixture of albumoses plus toxine rather than a toxic albumose, as Duclaux ${ }^{1}$ pointed out some few years back.

In his exhaustive chemical researches Martin has, as it seems, not realised the extensive distribution of the diphtheria bacillus in most, if not all, fatal cases. His own cases are just the kind of cases in which we should have expected to find bacilli in the lungs, for they were generally cases of laryngeal diphtheria (4 out of 6 ), and in one of these there was also broncho-pneumonia. But, anyhow, the observations of Wright and Stokes and our own show that in fatal cases of diphtheria the lung is almost always infected (namely, in 65 out of 66 cases).

We think, therefore, that the simplest view to take of the process of diphtheria intoxication is that the bacilli wherever present secrete a toxine-which is not an albumose, but a substance, at present chemically undefined-which is directly absorbed and acts directly as a nerve and general tissue poison, and that the albumoses are only secondary products, poisonous no doubt, but not of specific importance; and that since in fatal cases the bacilli are present in large numbers in the membranes, and are absent or present only in smaller numbers in the spleen, therefore a less active toxine is obtainable from the latter than from the former; and that when bacilli are absent from the spleen the toxine has been absorbed into the spleen from the membranes or lungs. At anyrate, it seems to us that the albumose theory, in the form proposed by Martin, cannot stand, unless the absence of bacilli beyond the membranes is established, and even this would hardly be a convincing argument.

2. Clinically we think our observations are of importance, since they prove the necessity of using the antitoxine energetically in all serious cases of diphtheria, the amount of toxine to be counteracted being always enormous when the bacilli have gained access to the lungs or other organs. The existence or suspicion of broncho-pneumonia should always excite us to action, and the antitoxine should not be spared when this complication arises. We would also suggest that in laryngeal cases prompt and copious injections should be administered in order to circumvent the dangers of a diphtheritic bronchopneumonia.

1 Ann. de l'Inst. Pasteur, Paris, 1892, tome vi. p. 369. 